\title{
Discovering Traditional Chinese Medicinal Herbs for Neurodegenerative Disorders
}

\author{
XUEWEI LIU, CAIXIA JIA, CHUANHONG WU², NA WANG, YONGMING JIA, YONGTAO ZHU^*, PENGYUE LI*, MURTAZA \\ GHULAM $^{3 *}$ AND KUNLIN ZHANG ${ }^{4,5 *}$ \\ College of Pharmacy, Qiqihar Medical University, Qiqihar, 161006, China, ${ }^{1}$ Beijing University of Chinese Medicine, Beijing, \\ 100029, ${ }^{2}$ The Biomedical Sciences Institute of Qingdao University (Qingdao Branch of SJTU Bio-X Institutes), Qingdao \\ University, Qingdao, 266071, ${ }^{3}$ Department of Pharmacy, COMSATS University Islamabad, Lahore Campus, Pakistan, ${ }^{4}$ Center \\ for Genetics and BioMedical Informatics Research, CAS Key Laboratory of Mental Health, Institute of Psychology, Chinese \\ Academy of Sciences, Beijing 100101, ${ }^{5}$ Department of Psychology, University of Chinese Academy of Sciences, Beijing \\ 100049, China
}

Liu et al.: Discovering TCM herbs for neurodegenerative disorders

\begin{abstract}
In this investigation, candidate traditional Chinese medicinal herbs for 3 neurodegenerative disorders, late-onset Alzheimer's disease, late-onset Parkinson's disease and Huntington disease were evaluated. The susceptibility genes were integrated to reconstruct traditional Chinese medicine-modern medicine network to discover the candidate herbs. For the 3 neurodegenerative disorders, the number of diseaserelated genes were increased from 2 to 16, which are directly associated with ingredients. Many traditional Chinese medicinal herbs associated with the 3 neurodegenerative disorders were identified (false discovery rate Benjamini-Hochberg< $<$.05). Four herbs for late-onset Alzheimer's disease are related to $\beta$-amyloid 1-42 measurement-associated genes BCAM and APOE by ingredients resveratrol and kainic acid. There are 3 herbs correlated to late-onset Parkinson's disease by both ingredient support and modern medicine symptom association evidence. Four traditional Chinese medicinal herbs are related to Huntington disease by ingredients. Available animal experiments in rats indicated that resveratrol could help to clean $\beta$-amyloid in brain to treat Alzheimer's disease, probably through targeting LRP1 protein. This study provided a novel way to utilize genetic data to explore traditional Chinese medicinal herbs and a new insight into drug discovery of neurodegenerative disorders.
\end{abstract}

Key words: Neurodegenerative disorders, susceptibility gene, traditional Chinese medicine herbs, Alzheimer's disease, resveratrol

Neurodegenerative disorders (NDs) are a group of diseases occurring because of progressively losing neuron structure or function (neurodegeneration). ND includes Alzheimer's disease (AD), Parkinson's disease (PD), Huntington disease (HD) and amyotrophic lateral sclerosis (ALS) ${ }^{[1]}$. AD can be divided into two categories, early-onset $\mathrm{AD}$ and late-onset $\mathrm{AD}$. Only a small proportion of $\mathrm{AD} / \mathrm{PD}$ patients carry causal mutation(s) of some single gene. For most patients, $\mathrm{AD} / \mathrm{PD}$ is caused by combined effects of genetic factor (multiple genes) and environmental triggers (common disease). Late-onset AD (LOAD, or sporadic AD) and late-onset PD (LOPD, or called sporadic PD) are typically such kinds of common diseases ${ }^{[2]}$. Traditional Chinese medicine (TCM) can protect and improve the brain, memory, and nervous system, but the exact ingredients or genes which are at work is not yet very clear. The reason why modern pharmaceutical scientists have difficulty to understand the knowledge of the symptoms traditionally treated by herbs is that it is hard to understand the definitions of TCM symptoms and exactly relate these to the symptoms used in modern medicine $(\mathrm{MM})^{[3]}$. Benefited from the research progresses of TCM, now it is possible to connect TCM herbs to disease through either ingredients and genes or TCM symptoms and MM symptoms. This situation has a potential top provide new insights in to drug discovery. In this study, an attempt has been made to integrate the ND susceptibility genes identified by genome-wide association study (GWAS) into TCM and MM data to reconstruct the TCM-MM network of 3 NDs (LOAD, LOPD and HD) to discover the TCM herbs related to NDs. All analyses of the present study were performed using Python 2.1.6 (https://www.python.org/) and R 3.4 .3 (https://cran.r-project.org/). For making herbdisease association, there are 2 paths, herb-ingredienttarget (gene)-disease and herb-TCM symptoms-MM symptoms-disease. These two paths correspond to

*Address for correspondence

E-mail: gmdogar356@gmail.com (Ghulam Murtaza); zhangkı@psych.ac.cn (Kunlin Zhang) 
2 drug discovery approaches, target-based drug discovery and phenotype-based drug discovery, respectively ${ }^{[4]}$. Information on the susceptibility genes of the 3 NDs was first collected, followed by TCM/MM components and finally the data on genes and TCM/MM was integrated to discover herb-disease association.

Three NDs (LOAD, LOPD and HD) were selected and the susceptibility genes (genes associated with these diseases) were collected for the NHGRI-EBI GWAS Catalog (v1.02, March 1, 2019) $)^{[5]}$. First the SNPs reaching genome-wide significance $(p$ value $<5 e-8$ ) were selected. For each selected SNP, if it has been mapped to some gene(s) in original research, those gene(s) were considered as susceptibility gene(s), otherwise the gene(s) mapped by GWAS Catalog were regarded as susceptibility gene(s). To obtain LOAD genes with functional support, the LOAD susceptibility genes that were also correlated to $\beta$-amyloid $1-42$ measurement ( $p$ value $<5 e-8$ ) were selected. The purpose of this study is to link the herbs to NDs by integrating newly-identified susceptibility genes into $\mathrm{TCM} / \mathrm{MM}$ data. The TCM/MM data collection, mainly the direct association between TCM/MM components, is based on the SymMap framework ${ }^{[3]}$. Briefly, the herbindigent association came from the TCM systems pharmacology (TCMSP), TCM integrated database (TCMID) and TCM information databases (TCM-ID); the ingredient-target (gene) associations were based on the herbal ingredients targets (HIT) and TCMSP databases; the gene-disease associations were extracted from the online Mendelian inheritance in man (OMIM) and human phenotype ontology (HPO) databases; herbTCM symptom associations were obtained directly from the CHPH with standardized TCM symptom terms; the TCM symptom-MM symptom mapping was built using an iterative process of experts' review; MM symptom-disease connections were based on the OMIM, HPO and Orphanet databases ${ }^{[6-12]}$. The susceptibility genes were first associated to the 3 NDs, followed by employing a two-step approach to detect herbs associated with diseases. The first step was to link the herbal ingredient to disease through genes. The second step was to detect herb-disease association through ingredient by Fisher's exact test. In detail, for a specific herb and a specific disease, 4 counts a, b, c and $d$ were calculated. Here $a$ is the number of ingredients directly link to both the herb and the disease, $b$ is the number of ingredients only directly linked to the herb, $\mathrm{c}$ is the number of ingredients only directly linked to the disease and $d$ is the number of ingredients directly linked to neither. These 4 counts formed a $2 \times 2$ contingency table and the Fisher's exact test was used to predict whether the herb is associated with the disease and $p$ value was generated. Then the false discovery rate (FDR) based on Benjamini-Hochberg (BH) method was used for multiple comparison correction ${ }^{[13,14]}$. The Q of FDR (BH) is the proportion of falsely rejected null hypotheses and the expectation of $\mathrm{Q}$ is FDR. The Benjamini-Hochberg method was chosen since it showed greater power in comparison to other multiple comparison correction methods such as family-wise error rate and Bonferroni test ${ }^{[15]}$. Here for FDR $(\mathrm{BH})$, the true null hypothesis is defined as that herb and disease are independent; the false null hypothesis is that herb and disease are correlated. Bonferroni-corrected $p$ value was also provided as a reference ${ }^{[16]}$. The herbdisease pairs with FDR $(\mathrm{BH})<0.05$ were kept. To investigate the properties and details of herb-disease association, herb-gene associations were also identified for the genes associated with the 3 NDs using similar methods. An alternative path for herb-disease through TCM symptom and MM symptom was also investigated by using similar method. The above analyses were performed for all the 3 NDs. In the screening of drugs for PD, to increase confidence, those herbs that can combine both herb-ingredient-target (gene)-disease path and herb-TCM symptom-MM symptom-disease path were considered. There were 13 susceptibility genes for LOAD, 118 susceptibility genes for LOPD and 6 susceptibility genes for HD, respectively (Table 1 and Supplementary Table 1). All the susceptibility genes, together with the 5 additional disease-related genes provided by SymMap (Table 1), were used to detect herb-disease association. For the 3 NDs, this increased the number of disease-related genes, which are directly associated with ingredients from 2 to 16 (in comparison to original SymMap). Based on the ingredient-disease associations and the herb-MM symptom association, 11 herb-disease associations for the 3 NDs were identified (Table 2). There were 4 herbs for LOAD, 3 for LOPD and 4 for HD. Most of herbdisease associations were newly identified based on susceptibility genes. The TCM-MM networks of the 3 NDs were shown in fig. 1. Radix Ginseng (Renshen, Ginseng), Folium Ilicis Cornutae (Gouguye), Herba Abri (Jigucao, Canton Love-pea Vine) and Caulis Piperis Kadsurae (Haifengteng) were considered to be the most potential treatments for LOAD, Fructus Gardeniae (Zhizi, fruit of Cape Jasmine), Morus alba L. (Sangshen, Mulberry Fruit) and Semen Pruni (Yuliren, Chinese Dwarf Cherry Seed) were considered to be the most potential treatments for LOPD and 
TABLE 1: SUMMARY OF SUSCEPTIBILITY GENES OF THE 3 NDS

\begin{tabular}{|c|c|c|c|c|c|c|}
\hline NDs & $\begin{array}{c}\text { Number of } \\
\text { susceptibility genes }\end{array}$ & \multicolumn{3}{|c|}{$\begin{array}{l}\text { No. of susceptibility genes with ingredient- } \\
\text { target information }\end{array}$} & \multicolumn{2}{|c|}{$\begin{array}{l}\text { No. of genes originally used } \\
\text { in SymMap }\end{array}$} \\
\hline $\begin{array}{l}\text { Late-onset Alzheimer's } \\
\text { disease (LOAD) }\end{array}$ & 13 & \multicolumn{3}{|c|}{2 (APOE, BCAM) } & \multicolumn{2}{|c|}{1 susceptibility gene (APOE) } \\
\hline $\begin{array}{l}\text { Late-onset Parkinson's } \\
\quad \text { disease (LOPD) }\end{array}$ & $\begin{array}{r}13(\mathrm{SC} \\
\mathrm{BAG} 3\end{array}$ & \multicolumn{3}{|c|}{$\begin{array}{c}13 \text { (SCARB2, STK39, TLR9, CTSB, SNCA, BAP1, } \\
\text { BAG3, DGKQ, GCH1, ALAS1, MAPT, COL3A1, } \\
\text { CRHR1) }\end{array}$} & \multicolumn{2}{|c|}{$\begin{array}{l}2 \text { non- susceptibility genes } \\
\text { (SNCAIP, NR4A2) }\end{array}$} \\
\hline $\begin{array}{l}\text { Huntington disease } \\
\text { (HD) }\end{array}$ & 6 & \multicolumn{3}{|c|}{1 (MSH3) } & \multicolumn{2}{|c|}{$\begin{array}{l}3 \text { non-susceptibility genes } \\
\text { (HTT, SLC2A3, TBX18) }\end{array}$} \\
\hline \multicolumn{7}{|c|}{${ }^{*}$ Only APOE and HTT are with ingredient-gene information } \\
\hline \multicolumn{2}{|r|}{ Herb $^{a}$} & Disease & p value & FDR(BH) & $\begin{array}{c}\text { FDR } \\
\text { (Bonferroni) }\end{array}$ & Relationship \\
\hline \multicolumn{2}{|c|}{ Gouguye, Folium Ilicis Cornutae } & LOAD & 0.013124 & 0.017498 & 0.052494 & By_ingredient \\
\hline \multicolumn{2}{|c|}{$\begin{array}{l}\text { Haifengteng, Caulis Piperis Kadsurae; Caulis Piperis } \\
\text { futokadsurae, stem of Kadsura Pepper }\end{array}$} & LOAD & 0.005606 & 0.011212 & 0.022424 & By_ingredient \\
\hline \multicolumn{2}{|c|}{ Jigucao, Herba Abri, Canton Love-pea Vine } & LOAD & 0.005402 & 0.011212 & 0.021609 & By_ingredient \\
\hline \multicolumn{2}{|c|}{ Renshen, Radix Ginseng, Ginseng } & LOAD & 0.023036 & 0.023036 & 0.092142 & By_ingredient \\
\hline \multicolumn{2}{|c|}{ Sangsheng, Fructus Mori, Mulberry Fruit } & LOPD & 0.000502 & 0.009443 & 0.151094 & By_ingredient \\
\hline \multicolumn{2}{|c|}{ Sangsheng, Fructus Mori, Mulberry Fruit } & LOPD & 0.00544 & 0.013955 & 0.320973 & By_MM_symptom \\
\hline \multicolumn{2}{|c|}{ Yuliren, Semen Pruni, Chinese Dwarf Cherry Seed } & LOPD & 0.000148 & 0.00637 & 0.044589 & By_ingredient \\
\hline \multicolumn{2}{|c|}{ Yuliren, Semen Pruni, Chinese Dwarf Cherry Seed } & LOPD & 0.000706 & 0.008517 & 0.041682 & By_MM_symptom \\
\hline \multicolumn{2}{|c|}{ Zhizi, Fructus Gardeniae, fruit of Cape Jasmine } & LOPD & 0.00964 & 0.042672 & 1 & By_ingredient \\
\hline \multicolumn{2}{|c|}{ Zhizi, Fructus Gardeniae, fruit of Cape Jasmine } & LOPD & 0.016072 & 0.028734 & 0.948228 & By_MM_symptom \\
\hline \multicolumn{2}{|c|}{$\begin{array}{l}\text { Baibiandou, Semen Lablab Album, White Hyacinth } \\
\text { Bean }\end{array}$} & $\mathrm{HD}$ & 0.009267 & 0.013439 & 0.037067 & By_ingredient \\
\hline \multicolumn{2}{|c|}{ Hetaoren, Semen Juglandis, English Walnut Seed } & HD & 0.010079 & 0.013439 & 0.040317 & By_ingredient \\
\hline \multicolumn{2}{|c|}{$\begin{array}{l}\text { Shanzhuyu, Fructus Corni, Asiatic Cornelian Cherry } \\
\text { Fruit }\end{array}$} & $\mathrm{HD}$ & 0.030185 & 0.030185 & 0.120742 & By_ingredient \\
\hline \multicolumn{2}{|c|}{ Suoluozi, Semen Aesculi, Buckeye Seed } & $\mathrm{HD}$ & 0.005911 & 0.013439 & 0.023645 & By_ingredient \\
\hline
\end{tabular}

Fructus Corni (Shanzhuyu, Asiatic Cornelian Cherry Fruit), Semen Aesculi (Suoluozi, Buckeye Seed), Semen Lablab Album (Baibiandou, White Hyacinth Bean) and Semen Juglandis (Hetaoren, English Walnut Seed) were considered to be the most potential treatment for HD. Through herb-ingredient-gene-disease path, 4 herbs were identified, Folium Ilicis Cornutae, Caulis Piperis Kadsurae, Herba Abri and Radix Ginseng (Table 2). The extended TCM-MM network by adding genes only associated with LOAD was shown in fig. 2 . Folium Ilicis Cornutae and Radix Ginseng were related to $B C A M$ (a target associated with both LOAD and $\beta$-amyloid 1-42 measurement) through the ingredient resveratrol and correlated to $B C H E$ (a modulator of cerebral amyloid deposition) through several ingredients (fig. 2). Caulis Piperis Kadsurae and Herba Abri were related to $A P O E$ (the other target associated with both LOAD and $\beta$-amyloid 1-42 measurement) through ingredient kainic acid (fig. 2). It has been reported that resveratrol can activate peroxisome proliferatorsactivated receptors (PPARs) family protein (PPAR $\alpha$,
PPAR $\beta$ and PPAR $\delta$ ) in vitro and in vivo ${ }^{[17]}$. PPARs can up-regulate gene and protein expression of LRP1, which is important for $\beta$-amyloid clearance ${ }^{[18]}$. For LOPD, 3 TCM herbs were significantly associated with LOPD by both ingredients and MM symptoms (Table 2). Morus alba L., Semen Pruni and Fructus Gardeniae were all have the function of moistening dryness, in terms of these results, these 3 herbs are associated with constipation in PD and might play a role in relieving constipation of $\mathrm{PD}$. In addition, the present investigation showed that Semen Pruni and Fructus Gardeniae were both linked to the ingredient ursolic acid and a susceptibility gene CTSB, the 3 herbs have a common active ingredient, quercetin and are related to the susceptibility gene COL3A1. According to a report quercetin is a polyphenol, which could change the progression of $\mathrm{ND}^{[9]}$. Quercetin could induce the activation of PKD1 and Akt in MN9D dopaminergic neuronal cells of MitoPark transgenic mouse model of $\mathrm{PD}$, moreover, it also could play a neuroprotective role by increasing mitochondrial bioenergetics capacity ${ }^{[10]}$. 

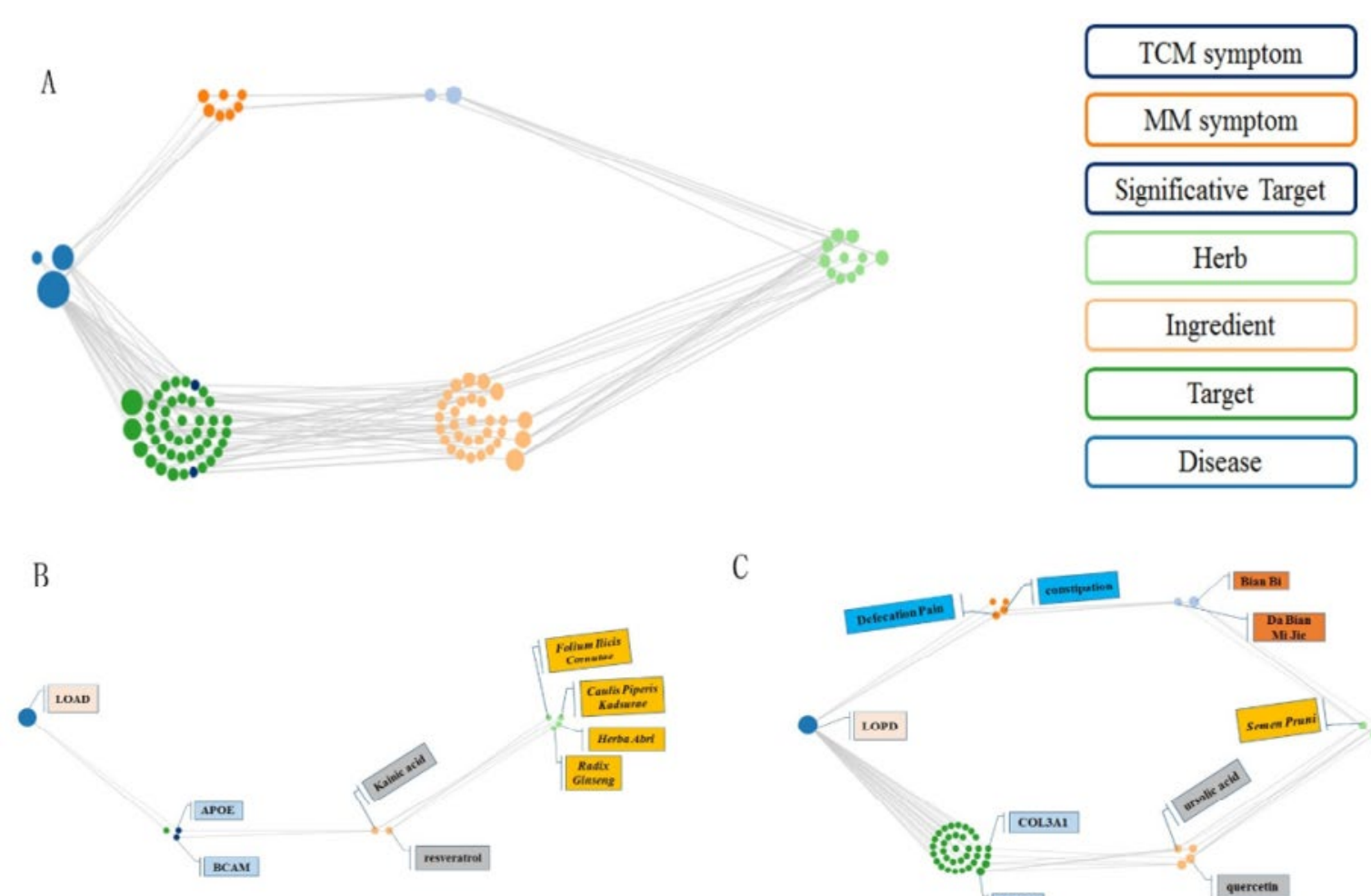

C

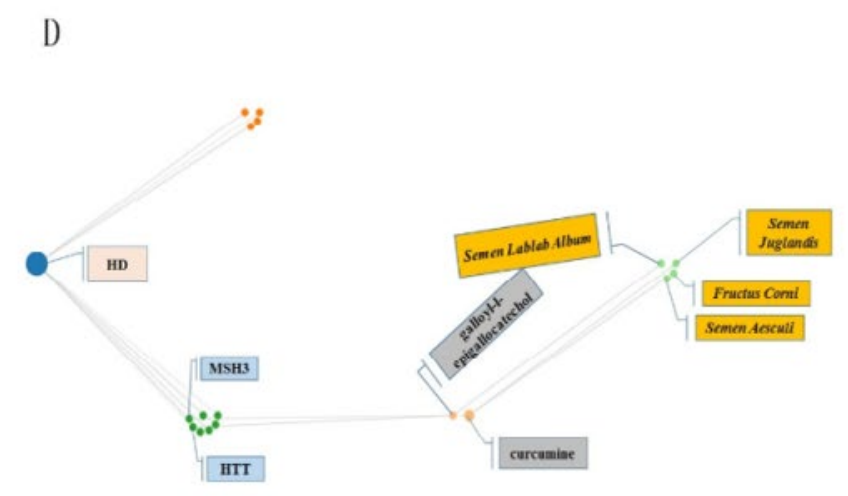

Fig. 1: TCM-MM networks for AD, LOPD and HD

TCM-MM networks of the 3 neurodegenerative disorders, late-onset Alzheimer's disease, late-onset Parkinson's disease and Huntington disease. The figure is for all the nodes and the nodes related to significant herbs with FDR $(\mathrm{BH})<0.05$ are in deeper colors. A) is for all the 3 NDs, B) is for LOAD, C) is for LOPD and D) is for HD.

COL3A1, which is involved in regulation of cortical development usually occurs in most soft connective tissues. There are reports to show that knockdown of COL $3 A 1$ suppressed the proliferation of neurofibroma cells it is possible that the effect of these 3 herbs on treating LOPD could be related to the quercetin on COL $3 A I^{[4]}$. There were 4 herbs identified to be related to $\mathrm{HD}$, through herb-ingredient-gene-disease path. They are Semen Lablab Album (Baibiandou, White Hyacinth Bean,), Semen Juglandis, Fructus Corni and Semen Aesculi. These 4 herbs can improve the condition of Huntington's patients by different methods. Present results showed that Semen Lablab Album might treat HD by galloyl-1-epigallocatechol, which is related to the susceptibility gene HTT. Semen Juglandis, Fructus Corni and Semen Aesculi have a common active ingredient curcumin which is related to the susceptibility gene MSH3. $\mathrm{AD}$ is a complex and multifactorial neurodegenerative disease, which brings great trouble to the life of patients. Among diverse types of $A D$, LOAD is more common ${ }^{[14]}$. AD belongs to the category of dementia syndrome under TCM. Symptoms often manifest as phlegm obstruction, verbal inversion, inappropriate behaviour and so on as is recorded in Jingyue's Complete Works (Jingyue Quanshu, Zhang Jingyue, Ming Dynasty) and Dialectical anecdotes (Chen Shiduo, Qing Dynasty) ${ }^{[5]}$. In TCM, dementia is related to weakness of spleen and stomach, deficiency 


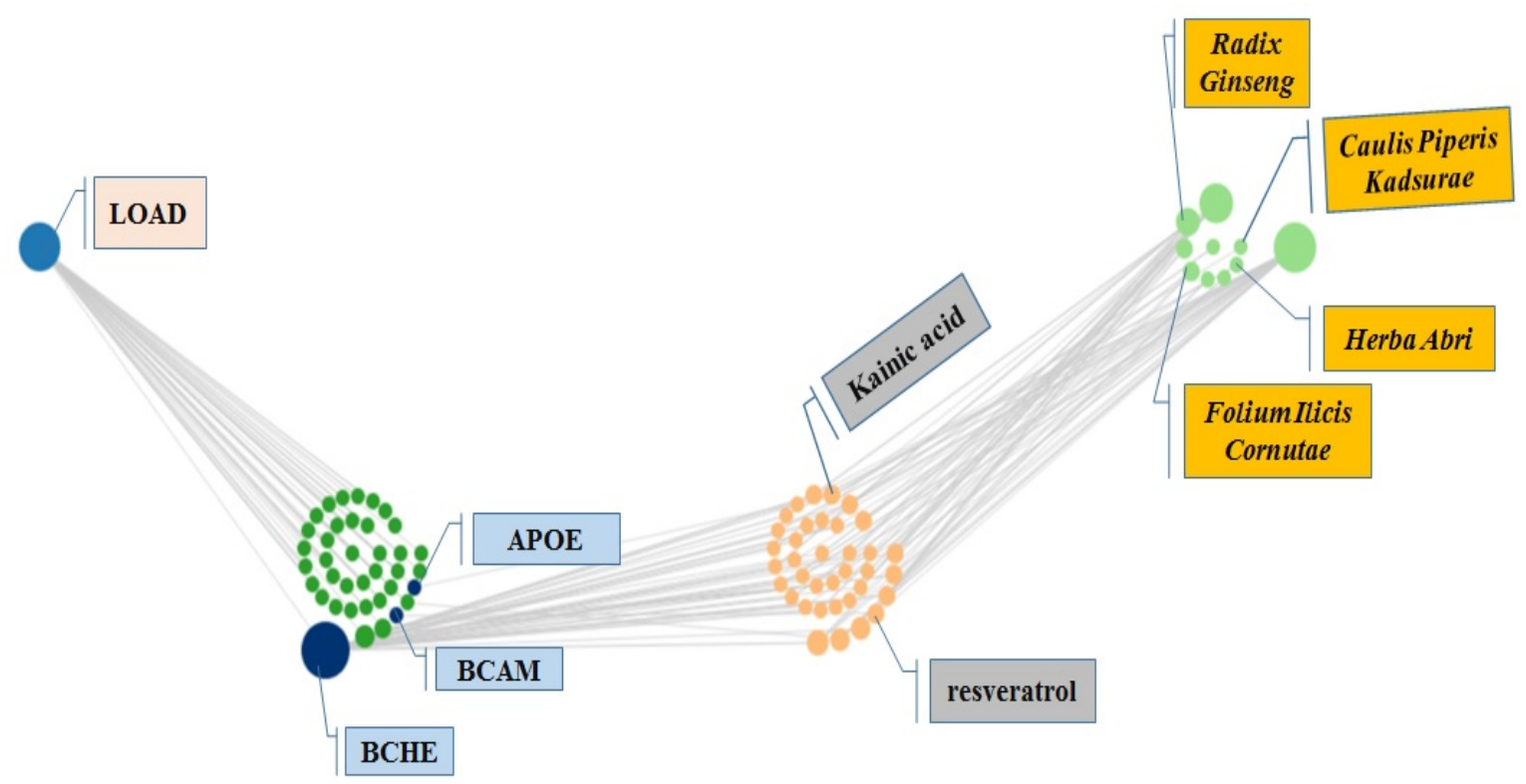

Fig. 2: The extended TCM-MM networks of the herbs for LOAD

of kidney essence, obstruction of phlegm and turbidity. Many researchers reported positive results in their dialectical treatment. At the beginning of the $20^{\text {th }}$ century, the hypothesis of pathogenesis of AD in TCM and the scheme of differentiating and treating $\mathrm{AD}$ by stages were put forward and preliminary clinical verification and approval were obtained ${ }^{[6]}$. According to scientific evidence, dementia is a progressive clinical manifestation that occurs due to depletion of acetylcholine due to degeneration of Ccholinergic nerves. Folium Ilicis Cornutae, Caulis Piperis Kadsurae, Herba Abri and Radix Ginseng were the TCM herbs screened in the present investigation; these have their own way of treating AD. For example, according to the theory of TCM, AD can be treated by the ingestion of kidney tonics. Yi Lin Sheng Mo mentioned that the evidence of forgetfulness is not from one end and the cause of the disease lies in the heart and kidney ${ }^{[8]}$. Huangdi's Canon of Medicine also said that the brain is sea of marrow and kidney stores essence to generate marrow ${ }^{[9]}$. If a person has insufficient kidney essence, the brain will not have enough nutrients and the brain-dominated life activities would be abnormal, such as abnormal memory and action. Shen Nong's herbal classic records that Folium Ilicis Cornutae has the effect of tonifying liver and kidney, which may be the way it treats AD. PD is the second most common ND. Pathologically, neuronal degeneration in PD is a selective dopaminergic neuron predominantly in the substantia nigra compact region. It has been recorded in Yuan Dynasty in China and belongs to the category of tremor syndrome and internal wind in TCM. The record is about $600 \mathrm{y}$ earlier than that in other countries. In the literature of TCM classics, most of its records are symptoms, mostly manifested as muscle weakness, stiffness, tremor and slow movement. It mainly caused by $y i n$ deficiency of liver and kidney, deficiency of $Q i$ and blood, deficiency of Yang and $q i$ and internal motion of liver and wind are all related to it. Kidney is the foundation of the 5 internal organs, which is closely related to brain and limbs. If the kidney $q i$ is sufficient, then the human intelligence is developed and the physical strength is high. Just as the plain question mentioned for Yang qi, essence is nourishing spirit, soft is nourishing tendons, deficiency of kidney yang lost in Wenxi, tendon and vein dystrophy and caused head shaking and limb tremor, tendon and vein constriction ${ }^{[6]}$. ZhengZhiGaiYao mentioned that tremor manifests hand and foot contractions, or flexion or extension. The person who was old and shimmy after the illness, holding the weak, was suitable for using Si Wu Tang with the addition of the herbs which are nourishing the blood to stop wind, such as Gentiana macrophylla Pall. (Qinjiao), Gastrodia elata Blume (Tianma), Morus alba L. (Sangshen), Astragalus mongholicus Bunge (Huangqi), Buthus martensii Karsch (Quanxie) and Clematis chinensis Osbeck (Weilingxian $)^{[7]}$. Thus, it can be seen that deficiency of kidney is an important cause of PD. Through herb-ingredient-gene-disease path and herb-TCM symptom-MM symptom-disease path, Morus alba L., Fructus Gardeniae, and Semen Pruni were thought to be the significantly associated with LOPD. Morus alba L. can tonify kidney to treat PD, beyond that modern studies showed that mulberry anthocyanin and resveratrol from Morus alba L. had good protective effect on nervous system, anthocyanin 
can enhance mitochondrial function, inhibit the death of PD dopaminergic cells and improve the condition of $\mathrm{PD}^{[8]}$. Internal motion of liver wind also leads to tremor. Plain Questions recorded that various wind syndromes manifested as convulsions and vertigo mostly belong to liver diseases; all symptoms of abrupt muscle and vein constriction and physical rigidity that cannot be flexed and stretched belong to the category of wind syndrome ${ }^{[6]}$. In addition, pathological products such as blood stasis and phlegm and non-motor symptoms such as constipation and pain are also often accompanied in the course of disease progression. Therefore, the treatment of PD can be referenced from these aspects to screen the potential of TCM. Semen Pruni could relax bowel and improve constipation in patients with PD. Fructus Gardeniae has the function of purgation, clear heat and sedation, which can regulate the immune system and metabolism of the body and promote the recovery of nerves. HD is an autosomal dominantneurodegenerative disease that is often manifested as dance-like involuntary movements, and cognitive impairment ${ }^{[9]}$. It is an inherited single gene disorder, but the progression of $\mathrm{HD}$ is associated with polygenic factor. Present study found that the TCM herbs associated with HD were Semen Lablab Album, Semen Juglandis, Fructus Corni and Semen Aesculi. According to the basic theory of TCM, Semen Juglandis and Fructus Corni is characterized as replenishing liver and kidney, it is reported Fructus corni polysaccharide can protect the hippocampal tissues of epileptic rats and Semen Juglandis was considered to be a potential herb for the prevention or treatment of PD which can significantly improve in PD-like movement impairment through inhibited depletion of striatal DA and its metabolites in vivo. In summary, Chinese medicine has a promising prospect in treating $\mathrm{NDs}^{[1-3]}$. NDs are a group of complex and multifactorial mental illness; they are not effective to use only the western medicine on the market now. TCM has a long history of development, many single herbs and herbal compounds have been proved that can be employed to treat NDs which are assumed to be correlated with kidney essence deficiency, turbid phlegm blocking upper orifices, stasis of blood, liver and wind internal movement. During the past $14 \mathrm{y}$, many susceptibility genes (the genes which are associated with specific trait and identified by statistical genetics approach such as genome-wide association study) for many traits including diverse disorders have been identified. Discovering function of these susceptibility genes become a challenge. By integrating newly-identified susceptibility genes of NDs into TCM-
MM network, many new penitential TCM herbs were identified for NDs, with solid TCM or MM evidences. These results provided new insight into TCM herb discovery for NDs. The framework of the study is a template, which could be extended to Chinese herb discovery for common diseases.

\section{Author Contributions}

Xuewei Liu, Caixia Jia, Ghulam Murtaza and Chuanhong $\mathrm{Wu}$ designed the research and wrote the manuscript. Na Wang and Yongming Jia were involved in data analyses. Kunlin Zhang, Pengyue Li, Yongtao Zhu supervised the research and revised the manuscript.

\section{Funding}

This study was supported by the National Natural Science Foundation of China (No. 31470070), the Fundamental Research Funds for the Central Universities (2018-JYB-XJQ-007), double first-class personnel department-Research Funds of Chen Jianxin Scientific Research Team (1000061020051).

\section{Conflict of Interest Statement}

The authors declare that the research was conducted in the absence of any commercial or financial relationships that could be construed as a potential conflict of interest.

\section{REFERENCES}

1. Liu C, Fang Y. New insights of poly (ADP-ribosylation) in neurodegenerative diseases:a focus on protein phase separation and pathologic aggregation. Biochem Pharmacol 2019;34:465471.

2. Almeida GC, Mirfakhar SF, Perdigao C, Burrinha T. Impact of late-onset Alzheimer's genetic risk factors on beta-amyloid endocytic production. Cell Mol Life Sci 2018;75:2577-89.

3. Wu YZF, Yang K. SymMap: An integrative database of traditional Chinese medicine enhanced by symptom mapping. Nucleic Acids Res 2019;47:1110-7.

4. Swinney DC. Phenotypic vs. target-based drug discovery for first-in-class medicines. Clin Pharmacol Ther 2013;93:299301.

5. Buniello A, MacArthur JAL, Cerezo M, Harris LW, Hayhurst J, Malangone C. The NHGRI-EBI GWAS Catalog of published genome-wide association studies, targeted arrays and summary statistics 2019. Nucleic Acids Res 2019;47:1005-12.

6. Ru J, Li P, Wang J, Zhou W, Li B, Huang C. TCMSP: A database of systems pharmacology for drug discovery from herbal medicines. J Cheminformatic 2014;6:13-8.

7. Huang L, Xie D, Yu Y, Liu H, Shi Y, Shi T. TCMID 2.0: A comprehensive resource for TCM. Nucleic Acids Res 2018;46:1117-20.

8. Chen X, Zhou H, Liu YB, Wang JF, Li H, Ung CY. Database of traditional Chinese medicine and its application to studies of mechanism and to prescription validation. Br J Pharmacol 2006;149:1092-103.

9. Ye H, Ye L, Kang H, Zhang D, Tao L, Tang K, et al. 
HIT:linking herbal active ingredients to targets. Nucleic Acids Res 2011;39:1055-9.

10. Hamosh ASAF, Amberger JS. Online Mendelian Inheritance in Man (OMIM), a knowledge base of human genes and genetic disorders. Nucleic Acids Res 2005;33:514-7.

11. Kohler S, Vasilevsky NA, Engelstad M, Foster E, McMurry J, Ayme S. The Human Phenotype Ontology in 2017. Nucleic Acids Res 2017;45:865-76.

12. Rath A, Olry A, Dhombres F, Brandt MM, Urbero B, Ayme S. Representation of rare diseases in health information systems: the Orphanet approach to serve a wide range of end users. Human Mutat 2012;33:803-8.

13. Fisher RA. On the Interpretation of $\chi 2$ from Contingency Tables, and the Calculation of P. J R Statist Soc 1922;85:87-94.

14. Benjamini Y, Hochberg Y. Controlling the false discovery rate: A practical and powerful approach to multiple testing. J R Statist Soc Ser B 1995;57:289-300.

15. Benjamini YHY. Controlling the false discovery rate: A practical and powerful approach to multiple testing. J R Statist Soc Ser B 1995;57:289-300.
16. Bland JM, Altman DG. Multiple significance tests: the Bonferroni method. Br Med J 1995;310:170-181.

17. Inoue H, Jiang XF, Katayama T, Osada S, Umesono K, Namura SJNL. Brain protection by resveratrol and fenofibrate against stroke requires peroxisome proliferator-activated receptor $\alpha$ in mice. Pak J Pharm Sci 2003;352:203-6.

18. Gauthier A, Vassiliou G, Benoist F, McPherson R. Adipocyte low density lipoprotein receptor-related protein gene expression and function is regulated by peroxisome proliferator-activated receptor gamma. J Biol Chem 2003;278:11945-53.

This is an open access article distributed under the terms of the Creative Commons Attribution-NonCommercial-ShareAlike 3.0 License, which allows others to remix, tweak, and build upon the work non-commercially, as long as the author is credited and the new creations are licensed under the identical terms

This article was originally published in a special issue, "Biomedical research applications in Pharmaceutical Sciences" Indian J Pharm Sci 2020:82(2)Spl issue3;70-76 